\title{
When Infinite Flow is Sufficient for Ergodicity
}

\author{
Behrouz Touri \\ Dept. of Industrial and Enterprise Systems Engineering \\ University of Illinois, Urbana, IL 61801 \\ Email: touri1@illinois.edu
}

\author{
Angelia Nedić \\ Dept. of Industrial and Enterprise Systems Engineering \\ University of Illinois, Urbana, IL 61801 \\ Email: angelia@illinois.edu
}

\begin{abstract}
We consider the consensus and ergodicity for a random linear discrete-time system driven by stochastic matrices. We focus on independent models with certain properties, and we study the ergodicity and consensus of such random models through a novel property, termed infinite flow property. Our key result is the establishment that for a class of independent random models, this property is a necessary and sufficient condition for ergodicity. Using this result, we show that the ergodicity of these models and the ergodicity of their expected models are the same. The result provides us with new tools for studying various aspects of dynamic networks and beyond. We demonstrate the potential use of our key result through several different applications. In particular, we apply it to provide a generalization of the randomized gossip algorithm and to study a consensus over a dynamic network with link failures. Also, we use the result to investigate necessary and sufficient conditions for the ergodicity of an equal-neighbor average algorithm on Erdös-Rényi random graphs. Finally, we demonstrate that our result can be employed to provide an alternative proof of the second Borel-Cantelli lemma.

Index Terms-Ergodicity, product of random stochastic matrices, linear random model, gossip algorithm, link failure, Borel-Cantelli lemma.
\end{abstract}

\section{INTRODUCTION}

The consensus and ergodicity over dynamic networks have been one of the central concepts studied in distributed control and optimization, as well as in the social and economic behavior literature. Due to the random nature of many applications, the study of those concepts on random models is important and challenging task. Most of the development of the subject is concentrated on the independently identically distributed (i.i.d.) models [9], [15], [8], [14] and stationary processes [10], [11], [6], [16]. In this paper, we focus on general random models and we develop some necessary conditions for the ergodicity of these models. Moreover, we show that the conditions are also sufficient for certain types of independent random models that are not necessarily identically distributed. To some extent, our work builds on the insights given in the early work of Tsitsiklis [18].

In Section II, we describe a class of random models, and we formulate the consensus and ergodicity concepts for these models. We also introduce the infinite flow property which plays a central role in all the subsequent development. In Section III, we show that the infinite flow property

This research was supported in parts by the National Science Foundation under CAREER grant CMMI 07-42538 and by the AFOSR under grant FA9550-09-1-0612. is a necessary condition for ergodicity in any random or deterministic model. We also show that the ergodicity of the expected model is a necessary condition for the ergodicity of the original random model for the class of independent random models. In Section IV, we define a feedback property and the class of models with a common steady state in expectation, and we investigate some properties of such models. We provide the fundamental result of this paper, the Infinite Flow Theorem in Section V. In Section VII, we investigate several applications of the Infinite Flow Theorem including an extension of the randomized gossip algorithm, link failure models and the random neighbor average algorithms on random graphs. Finally, we provide an alternative proof of the second Borel-Cantelli lemma by relating the infinitely often event of a sequence of random events with the ergodicity of a certain random model.

Notation and Basic Terminology. We use subscripts for indexing elements of vectors and matrices. We write $x \geq 0$ or $x>0$ if, respectively, $x_{i} \geq 0$ or $x_{i}>0$ for all $i$. We use $e_{i}$ to denote the $i$ th vector in the standard basis of $\mathbb{R}^{m}$ and $e$ to denote the vector with all entries equal to one. A vector $a$ is stochastic if $a \geq 0$ and $\sum_{i} a_{i}=1$. A matrix $W$ is stochastic if all of its rows are stochastic vectors, and it is doubly stochastic if its rows and columns are stochastic vectors. We use $[m]$ to denote the set $\{1, \ldots, m\}$. We denote a proper subset of $[m]$ by $S \subset[m]$ and its complement by $\bar{S}$. A set $S \subset[m]$ such that $S \neq \emptyset$ is a nontrivial subset of $[m]$. For a given $m \times m$ matrix $W$, we let $W_{S}=\sum_{i \in S, j \in \bar{S}}\left(W_{i j}+W_{j i}\right)$. We write $\mathrm{E}[X]$ to denote the expected value of a random variable $X$. For an event $\mathscr{A}$, we use $\operatorname{Pr}(\mathscr{A})$ to denote its probability. We often use a.s. to denote almost surely.

\section{Problem Formulation And Terminology}

Let $\mathbb{S}^{m}$ be the set of $m \times m$ stochastic matrices, and let $\mathscr{F}_{\mathbb{S}^{m}}$ denote the Borel sigma algebra on $\mathbb{S}^{m}$. For an arbitrary probability space $(\Omega, \mathscr{E}, \operatorname{Pr}(\cdot))$, a measurable function $W$ : $(\Omega, \mathscr{E}, \operatorname{Pr}(\cdot)) \rightarrow \prod_{k=0}^{\infty}\left(\mathbb{S}^{m}, \mathscr{F}_{\mathbb{S} m}\right)$ is termed a random chain, random dynamic or random model, and it is represented by the sequence of its coordinate maps $\{W(k)\}$. A random model $\{W(k)\}$ is an independent model if its coordinate maps $\{W(k)\}$ are independent, and it is an i.i.d. model if, in addition, its coordinate maps are identically distributed.

Given a deterministic chain $\{A(k)\}$, we define $\Phi(k, s)=$ $A(k) \cdots A(s-1) A(s)$ and consider the following definitions of ergodicity and consensus for the chain. The chain $\{A(k)\}$ 
admits consensus if $\lim _{k \rightarrow \infty} \Phi(k, 0)=e c^{T}$ for some $c \in$ $\mathbb{R}^{m}$. The chain is ergodic if $\lim _{k \rightarrow \infty} \Phi(k, t)=e c_{t}^{T}$ for any $t \geq 0$ and some $c_{t} \in \mathbb{R}^{m}$. Based on these definitions, we say that a random model $\{W(k)\}$ admits consensus if $\{W(k)(\omega)\}$ admits consensus for almost all realizations of $\omega$. Similarly, we say that the model $\{W(k)\}$ is ergodic if $\{W(k)(\omega)\}$ is ergodic for almost all realizations of $\omega$.

The study of both the consensus and ergodicity concepts of a chain is closely related to the study of a linear dynamic system driven by the chain. More precisely, for a given random chain $\{W(k)\}$, an initial time $t \geq 0$ and an initial vector $x(t) \in \mathbb{R}^{m}$ at time $t$, we will consider the following dynamics:

$$
x(k+1)=W(k) x(k) \quad \text { for } k \geq t .
$$

This dynamics defines a sequence $\{x(k)\}$ of random vectors We will frequently use the dynamic system (1) with a deterministic chain $\{A(k)\}$ (corresponding to a constant random chain $\{W(k)(\omega)\}=\{A(k)\})$, which results in a sequence of deterministic vectors $\{x(k)\}$.

If a deterministic chain is ergodic, then by definition for any $t \geq 0, \lim _{k \rightarrow \infty} \Phi(k, t)=e c_{t}^{T}$ and hence, for any $x(t) \in \mathbb{R}^{m}, \lim _{k \rightarrow \infty} x(k)=\lim _{k \rightarrow \infty} \Phi(k, t) x(t)=$ $\left(c_{t}^{T} x(t)\right) e$. Therefore, for any $i, j \in[m], \lim _{k \rightarrow \infty}\left(x_{i}(k)-\right.$ $\left.x_{j}(k)\right)=0$. The converse of this statement is also true in a stronger sense: if $\lim _{k \rightarrow \infty}\left(x_{i}(k)-x_{j}(k)\right)=0$, for any starting time $t \geq 0$, and $x(t)=e_{\ell}$ where $\ell \in[m]$, then $\lim _{k \rightarrow \infty}\left([\Phi(k, t)]_{\ell i}-[\Phi(k, t)]_{\ell j}\right)=0$. This condition is equivalent to weak ergodicity which is known to be equal to the (strong) ergodicity of the chain for stochastic matrices [4]. In order to prove that a (random) chain is ergodic, it suffice to study the behavior of the (random) sequence $x(k)$ driven by dynamic system (1) for starting point equals to $e_{\ell}$ for $\ell \in[m]$.

To show the ergodicity of particular models, we extensively use a phenomena referred to as infinite flow property. A chain $\{A(k)\}$ is said to have the infinite flow property if $\sum_{k=0}^{\infty} A_{S}(k)=\infty$ for any nontrivial set $S \subset[m]$.

\section{NECESSARY CONDITIONS FOR ERGODICITY}

In this section we establish two results on necessary conditions for the ergodicity of random models. The first result shows that the infinite flow property is necessary for the ergodicity of any (not necessarily independent) random model. The second result shows that, for independent random models, the ergodicity of the expected chain of a random chain is necessary for the ergodicity of the random chain.

For a sequence $\{x(k)\}$ and a nontrivial subset $S \subset[m]$, we define $d_{S}(k)=\min _{j \in \bar{S}} x_{j}(k)-\max _{i \in S} x_{i}(k)$. Using this definition, we have the following statement.

Lemma 1: Let $\{A(k)\}$ be a deterministic chain, and let $\{x(k)\}$ be generated by dynamic system (1) with an initial vector $x(0) \in \mathbb{R}^{m}$. Then, for any nontrivial set $S \subset[m]$ and $k \geq 0$, we have $d_{S}(k+1) \geq d_{S}(0)-2 d(x(0)) \sum_{t=0}^{k} A_{S}(t)$, where $d(y)=\max _{\ell \in[m]} y_{\ell}-\min _{r \in[m]} y_{r}$ for $y \in \mathbb{R}^{m}$.

Proof: Let $S \subset[m]$ be arbitrary. Let $x_{\min }(k)=$ $\min _{r \in[m]} x_{r}$ and $x_{\max }(k)=\max _{s \in[m]} x_{s}(k)$. By the stochasticity of $A(k)$ we have $x_{i}(k) \in\left[x_{\min }(0), x_{\max }(0)\right]$ for all $i \in[m]$ and all $k$. By the definition of $x(k+1)$ in Eq. (1) for any $i \in S$ we have,

$$
\begin{aligned}
& x_{i}(k+1)=\sum_{\ell \in S} A_{i \ell}(k) x_{\ell}(k)+\sum_{\ell \in \bar{S}} A_{i \ell}(k) x_{\ell}(k) \\
& \leq \sum_{\ell \in S} A_{i \ell}(k) \max _{s \in S} x_{s}(k)+x_{\max }(0) \sum_{\ell \in \bar{S}} A_{i \ell}(k) \\
& \leq\left(1-\sum_{\ell \in \bar{S}} A_{i \ell}(k)\right) \max _{s \in S} x_{s}(k)+x_{\max }(0) \sum_{\ell \in \bar{S}} A_{i \ell}(k) \\
& =\max _{s \in S} x_{s}(k)+\left(x_{\max }(0)-\max _{s \in S} x_{s}(k)\right) \sum_{\ell \in \bar{S}} A_{i \ell}(k),
\end{aligned}
$$

where the last inequality holds because of the stochasticity of $A(k)$. Note that $0 \leq \sum_{\ell \in \bar{S}} A_{i \ell}(k) \leq A_{S}(k)$ and $d(x(0)) \geq$ $x_{\max }(0)-\max _{s \in S} x_{s}(k) \geq 0$ and hence for any $i \in S$,

$$
x_{i}(k+1) \leq \max _{s \in S} x_{s}(k)+d(x(0)) A_{S}(k) .
$$

By taking the maximum over all $i \in S$ in the preceding relation and using the induction on $k$, we can show that

$$
\max _{i \in S} x_{i}(k+1) \leq \max _{s \in S} x_{s}(0)+d(x(0)) \sum_{t=0}^{k} A_{S}(t) .
$$

Now by using the above inequality for $\{\bar{x}(k)\}$ driven by (1) and $\bar{x}(0)=-x(0)$, and by exchanging the node elements of $S$ and $\bar{S}$, we would have $\max _{j \in \bar{S}} \bar{x}_{j}(k+1) \leq$ $\max _{s \in \bar{S}} \bar{x}_{s}(0)+d(\bar{x}(0)) \sum_{t=0}^{k} A_{S}(t)$. Since the dynamic system (1) is linear $\bar{x}(k)=-x(k)$, and it follows that $d(\bar{x}(0))=d(x(0))$. Furthermore,

$$
\begin{aligned}
-\min _{j \in \bar{S}} & x_{j}(k+1)=\max _{j \in \bar{S}} \bar{x}_{j}(k+1) \\
& \leq \max _{s \in \bar{S}} \bar{x}_{s}(0)+d(\bar{x}(0)) \sum_{t=0}^{k} A_{S}(t) \\
& \leq-\min _{s \in \bar{S}} x_{s}(0)+d(x(0)) \sum_{t=0}^{k} A_{S}(t) .
\end{aligned}
$$

By using the definition $d_{S}(x(k))=\min _{j \in \bar{S}} x_{j}(k)-$ $\max _{i \in S} x_{i}(k)$, and adding both sides of inequalities (2) and (3), we obtain the desired inequality.

The necessity of the infinite flow for any ergodic chain is a consequence of Lemma 1, as seen in the following result.

Theorem 1: Let $\{A(k)\}$ be a deterministic ergodic chain. Then $\sum_{k=0}^{\infty} A_{S}(k)=\infty$ for any nontrivial $S \subset[m]$.

Proof: Assume that the statement is not true and there is a nontrivial set $S \subset[m]$ such that $\sum_{k=0}^{\infty} A_{S}(k)<\infty$. Since the matrices $A(k)$ are stochastic, we have $A_{S}(k) \geq 0$ for all $k$. Therefore, there exists large enough $\bar{t} \geq 0$ such that $\sum_{k=\bar{t}}^{\infty} A_{S}(k)<\frac{1}{4}$.

Now, define the vector $\bar{z}=\left(\bar{z}_{1}, \ldots, \bar{z}_{m}\right)^{T}$ with $\bar{z}_{i}=0$ if $i \in S$ and $z_{i}=1$ if $i \in \bar{S}$ and consider the dynamic system in (1) with starting time $\bar{t}$ and $z(t)=\bar{z}$. Note that Lemma 1 can be applied to the case where the time $t=\bar{t}$ is taken as initial time. Since $d(\bar{t})=1$, by applying Lemma 1 , we have 
for all $k>\bar{t}$

$d_{S}(k) \geq d_{S}(\bar{t})-2 d_{S}(\bar{t}) \sum_{t=\bar{t}}^{k} A_{S}(t) \geq 1-2 \sum_{t=\bar{t}}^{\infty} A_{S}(t) \geq \frac{1}{2}$,

implying that the chain $\{A(k)\}$ is not ergodic - a contradiction. Hence, $\{A(k)\}$ must have the infinite flow property.

Theorem 1 holds for any random model. The following lemma gives another necessary condition for ergodicity of any independent random model.

Lemma 2: Let $\{W(k)\}$ be an independent model. Then, the consensus (ergodicity) of the expected chain $\{\mathrm{E}[W(k)]\}$ is necessary for the consensus (ergodicity) of the chain.

Proof: Consider $\{x(k)\}$ defined by (1) with any initial state $x(0)$. Since each $\{W(k)\}$ is stochastic, we have $\min _{\ell} x_{\ell}(0) \leq x_{i}(k) \leq \max _{j} x_{j}(0)$ for all $k \geq 0$ and $i \in[m]$. By the dominated convergence theorem, it follows that $\mathrm{E}\left[\lim _{k \rightarrow \infty} x(k)\right]=\lim _{k \rightarrow \infty} \mathrm{E}[x(k)]$.

Suppose now that the consensus event occurs a.s. for the chain $\{W(k)\}$. Then, $\lim _{k \rightarrow \infty} x(k)=$ ce a.s. for some random $c \in \mathbb{R}$, and $\lim _{k \rightarrow \infty} \mathrm{E}[x(k)]=\mathrm{E}[c] e$. On the other hand, since the model is independent, it follows that $\mathrm{E}[x(k)]=\mathrm{E}[W(k-1)] \cdots \mathrm{E}[W(0)] x(0)$, implying that

$$
\lim _{k \rightarrow \infty} \mathrm{E}[W(k-1)] \cdots \mathrm{E}[W(0)] x(0)=\mathrm{E}[c] e .
$$

Hence, consensus is reached in the expected chain.

When the ergodicity occurs a.s., we apply the preceding argument for any starting time $T$ instead of time 0 , and we obtain that the expected chain is ergodic.

\section{Random Models with FeEdback Property And STEAdy State IN ExPECTATION}

We consider the random models with two properties: weak feedback property and a common steady state in expectation. The former property is defined as follows.

Definition 1: (Weak Feedback Property) A random model $\{W(k)\}$ is said to have weak feedback property if there exists $\gamma>0$ such that

$$
\mathrm{E}\left[\left(W^{i}(k)\right)^{T} W^{j}(k)\right] \geq \gamma\left(\mathrm{E}\left[W_{i j}(k)\right]+\mathrm{E}\left[W_{j i}(k)\right]\right)
$$

for all $k \geq 0$, and all $i, j \in[m]$ with $i \neq j$, where $W^{i}$ denotes the $i$ th column vector of a matrix $W$. The scalar $\gamma$ is referred to as the feedback constant.

A class of models with weak feedback property is, for example, the class of random (and deterministic) models $\{W(k)\}$ that have the diagonal entries of $W(k)$ strictly greater than a constant $\gamma>0$ almost surely for all $k \geq 0$. Such models have been studied in [18], [19], [13], [12]. Also, as shown in [17], the weak feedback property is inherent in the i.i.d. models with positive diagonal entries that have been studied in [15].

Definition 2: A random model has a common steady state in expectation if there is a stochastic vector $\pi \in \mathbb{R}^{m}$ such that $\pi^{T} \mathrm{E}[W(k)]=\pi^{T}$ for all $k$.

For example, the class of i.i.d. random models have a common steady state in expectation. This holds since, for i.i.d. models, $\mathrm{E}[W(k)]$ does not depend on $k$ and such a vector $\pi$ exists since $\mathrm{E}[W(k)]$ is a stochastic matrix. Another class of such random models is the class of symmetric random models in expectation i.e., $\{W(k)\}$ with $\mathrm{E}\left[W_{i j}(k)\right]=$ $\mathrm{E}\left[W_{j i}(k)\right]$ for any $k \geq 0$ and $i, j \in[m]$. For such models, by the symmetric property and the stochasticity of each $W(k)$, the model has the common steady state $\pi=\frac{1}{m} e$ in expectation. In Section VII, we will visit some applications dealing with the symmetric random models.

As in the case of deterministic chains, the function $L(x)=$ $\sum_{i=1}^{m} \pi_{i}\left(x_{i}-\pi^{T} x\right)^{2}$, defined for $x \in \mathbb{R}^{m}$, is important for studying the chains with a common steady state $\pi$ in expectation. The following result is essential for the proofs of our results in Sections $\mathrm{V}$ and VI.

Theorem 2: Let the random model $\{W(k)\}$ be independent with a common steady state $\pi$ in expectation, and let $V_{k}=L(x(k))$ for all $k$. Then, for all $k \geq 0$ we almost surely have $\mathrm{E}\left[V_{k+1} \mid x_{k}\right] \leq V_{k}-\sum_{i<j} H_{i j}(k)\left(x_{i}(k)-x_{j}(k)\right)^{2}$, where $H(k)=\mathrm{E}\left[W^{T}(k) D W(k)\right]$ and $D=\operatorname{diag}(\pi)$.

The proof of Theorem 2 is given in [17].

\section{SUFFICIENCY OF INFINITE FLOW}

Here, we prove that infinite flow is sufficient for ergodicity of random models with weak feedback property and a common steady state $\pi>0$ in expectation. To do so, we use Theorem 2 and the following lemma.

Lemma 3: Let $\{A(k)\}$ be a deterministic chain with infinite flow property, and let $z(k)=A(k-1) z(k-1)$ for $k \geq 1$ and $z(0)=e_{\ell}$ for some $\ell \in[m]$. Assume that $\lim _{k \rightarrow \infty}\left(z_{\max }(k)-z_{\min }(k)\right)>0$. Then,

$$
\sum_{k=0}^{\infty} \sum_{i<j}\left(A_{i j}(k)+A_{j i}(k)\right)\left(z_{i}(k)-z_{j}(k)\right)^{2}=\infty .
$$

Proof: Let $d_{k}=z_{\max }(k)-z_{\min }(k)$ and $d_{\infty}=$ $\lim _{k \rightarrow \infty}\left(z_{\max }(k)-z_{\min }(k)\right)>0$. For $t \geq 0$, let $\sigma$ (which is a function of $t$ ) be a permutation on $[m]$ such that $z_{\sigma_{1}}(t) \leq$ $\cdots \leq z_{\sigma_{m}}(t)$. Since the matrices $\{A(k)\}$ are stochastic, the sequence $\{d(k)\}$ is non-increasing and hence,

$$
d_{t}=z_{\sigma_{m}}(t)-z_{\sigma_{1}}(t)=\sum_{i=1}^{m-1}\left(z_{\sigma_{i+1}}(t)-z_{\sigma_{i}}(t)\right) \geq d_{\infty}
$$

Therefore, there exists an index $i^{*}$ such that $z_{\sigma_{i^{*}+1}}(t)-$ $z_{\sigma_{i^{*}}}(t) \geq \frac{1}{m-1} d_{\infty}$. Let $S=\left\{\sigma_{i} \mid i \leq i^{*}\right\}$, so that $d_{S}(t) \geq$ $\frac{1}{m-1} d_{\infty}$. Now, let $t^{\prime}=\arg \min _{s>t} \sum_{k=t}^{s-1} A_{S}(k) \geq \frac{1}{3} d_{S}(t)$. Then, by Lemma 1 , for $s \in\left[t, t^{\prime}\right]$, we have $d_{S}(s) \geq d_{S}(t)-$ $2 d(z(t)) \sum_{k=t}^{t^{\prime}} A_{S}(k) \geq \frac{1}{3} d_{S}(t)$. Since $d_{S}(t) \geq \frac{1}{m-1} d_{\infty}$, 
we have $d_{S}(s) \geq \frac{1}{3(m-1)} d_{\infty}$. Therefore,

$$
\begin{aligned}
\sum_{k=t}^{t^{\prime}-1} & \sum_{i<j}\left(A_{i j}(k)+A_{j i}(k)\right)\left(z_{i}(k)-z_{j}(k)\right)^{2} \\
& \geq \sum_{k=t}^{t^{\prime}-1} \sum_{i \in S, j \in \bar{S}}\left(A_{i j}(k)+A_{j i}(k)\right)\left(z_{i}(k)-z_{j}(k)\right)^{2} \\
& \geq \sum_{k=t}^{t^{\prime}-1} \sum_{i \in S, j \in \bar{S}}\left(A_{i j}(k)+A_{j i}(k)\right) d_{S}^{2}(k) \\
& =\sum_{k=t}^{t^{\prime}-1} d_{S}^{2}(k) \sum_{i \in S, j \in \bar{S}}\left(A_{i j}(k)+A_{j i}(k)\right)
\end{aligned}
$$

where the last inequality holds since $\left(z_{j}(k)-z_{i}(k)\right) \geq d_{S}(k)$ for $i \in S$ and $j \in \bar{S}$. Also note that by the definition $A_{S}(k)=\sum_{i \in S, j \in \bar{S}}\left(A_{i j}(k)+A_{j i}(k)\right)$. Therefore, we have

$$
\begin{aligned}
& \sum_{k=t}^{t^{\prime}-1} \sum_{i<j}\left(A_{i j}(k)+A_{j i}(k)\right)\left(z_{i}(k)-z_{j}(k)\right)^{2} \\
& \quad \geq \sum_{k=t}^{t^{\prime}-1} A_{S}(k) d_{S}^{2}(k) \geq\left(\frac{1}{3(m-1)} d_{\infty}\right)^{2} \sum_{k=t}^{t^{\prime}-1} A_{S}(k) \\
& \quad \geq\left(\frac{1}{3(m-1)} d_{\infty}\right)^{3}=\frac{1}{27(m-1)^{3}} d_{\infty}^{3}
\end{aligned}
$$

The preceding argument holds for arbitrary $t \geq 0$. Hence, by letting $t_{0}=0$ and recursively defining $t_{s}=t_{s-1}^{\prime}$ for $s>0$, we have

$$
\begin{aligned}
\sum_{k=0}^{\infty} & \sum_{i<j}\left(A_{i j}(k)+A_{j i}(k)\right)\left(z_{i}(k)-z_{j}(k)\right)^{2} \\
& =\sum_{s=0}^{\infty} \sum_{k=t_{s}}^{t_{s+1}-1} \sum_{i<j}\left(A_{i j}(k)+A_{j i}(k)\right)\left(z_{i}(k)-z_{j}(k)\right)^{2} \\
& \geq \sum_{s=0}^{\infty} \frac{1}{27(m-1)^{3}} d_{\infty}^{3}=\infty
\end{aligned}
$$

where the last inequality follows from Eqs. (4) and (5).

Based on Lemma 3 and Theorem 2, we have the following important result.

Theorem 3: Let $\{W(k)\}$ be an independent random model with a common steady state $\pi>0$ in expectation and feedback property with constant $\gamma>0$. Then, if the model has infinite flow property, the model is ergodic.

Proof: Since $\pi$ is a common steady state in expectation, by Theorem 2 we have for any $x(0) \in \mathbb{R}^{m}$,

$$
\sum_{k=0}^{\infty} \sum_{i<j} H_{i j}(k) \mathrm{E}\left[\left(x_{i}(k)-x_{j}(k)\right)^{2}\right]<\infty .
$$

Since $\pi>0$ we have $H_{i j}(k) \geq \pi_{\min } \mathrm{E}\left[W^{i}(k) W^{j}(k)\right]$, where $\pi_{\min }=\min _{i} \pi_{i}$. Due to the feedback property, we have $\mathrm{E}\left[W^{i}(k) W^{j}(k)\right] \geq \gamma \mathrm{E}\left[W_{i j}(k)+W_{j i}(k)\right]$. Therefore using the independency of the model and the above observations, for any $x(0) \in \mathbb{R}^{m}$, we have

$$
\begin{aligned}
& \sum_{k=0}^{\infty} \sum_{i<j} \mathrm{E}\left[\left(W_{i j}(k)+W_{j i}(k)\right)\left(x_{i}(k)-x_{j}(k)\right)^{2}\right] \\
& =\mathrm{E}\left[\sum_{k=0}^{\infty} \sum_{i<j}\left(W_{i j}(k)+W_{j i}(k)\right)\left(x_{i}(k)-x_{j}(k)\right)^{2}\right]<\infty,
\end{aligned}
$$

where the equality holds because of the non-negativeness of $\left(W_{i j}(k)+W_{j i}(k)\right)(k)\left(x_{i}(k)-x_{j}(k)\right)^{2}$ and monotone convergence theorem. As a result $\sum_{k=0}^{\infty} \sum_{i<j}\left(W_{i j}(k)+\right.$ $\left.W_{j i}(k)\right)(k)\left(x_{i}(k)-x_{j}(k)\right)^{2}<\infty$ almost surely for any $x(0)$. Hence, based on Lemma 3, we have $\lim _{k \rightarrow \infty}\left(x_{\max }(k)-\right.$ $\left.x_{\min }(k)\right)=0$ almost surely for $x(0)=e_{\ell}$ and $\ell \in[m]$, implying that the system reaches consensus almost surely. Since $\sum_{k=t}^{\infty} \sum_{i<j} H_{i j}(k) \mathrm{E}\left[\left(x_{i}(k)-x_{j}(k)\right)^{2}\right]<\infty$ for any starting time $t \geq 0$, by the same argument, it follows that the model is ergodic.

\section{NECESSARY AND SUFFICIENT CONDITION FOR ERGODICITY}

We now establish our main result of this paper, which is termed Infinite Flow Theorem due to the central role of the infinite flow property in its derivation.

Theorem 4: (Infinite Flow Theorem) Let $\{W(k)\}$ be an independent random model with expected steady state $\pi>0$ and weak feedback property. Then, the following conditions are equivalent:

(a) The model is ergodic.

(b) The expected model is ergodic.

(c) The expected model has infinite flow property.

(d) The model has infinite flow property.

Proof: If the model is ergodic, then by Lemma 2 the expected model is ergodic and hence, (a) implies (b). By Theorem 1, if the expected model is ergodic, then it must have infinite flow and hence, (b) implies (c). Since the model is independent, $\left\{W_{S}(k)\right\}$ is an independent sequence for all $S \subset[m]$. Also, since $0 \leq W_{S}(k) \leq m$ almost surely, by Kolmogorov's three series Theorem [7], page 63, for $\sum_{k=0}^{\infty} W_{S}(k)<\infty$ to hold a.s., it is necessary to have $\sum_{k=0}^{\infty} \mathrm{E}\left[W_{S}(k)\right]<\infty$. Thus, (c) implies (d). Finally by Theorem 3, (d) implies (a).

Since symmetric models have a common steady state $\frac{1}{m} e$ in expectation, the Infinite Flow Theorem applies to any independent symmetric random model with the weak feedback property. As discussed later in Section VII, Infinite Flow Theorem provides a powerful tool for studying various timechanging random systems.

Although the main advantage of the Infinite Flow Theorem is its applicability to time-changing random systems, it also provides some new insights for i.i.d. random models. Consider an i.i.d. random model $\{W(k)\}$. Due to the stochasticity and time-independency of the expected chain, any such model has a common steady state $\pi$ in expectation. Tahbaz-Salehi and Jadbabaie proved in [15] that any i.i.d. model with almost surely positive diagonal entries is ergodic 
if and only if the expected model is ergodic. The same result follows from our Infinite Flow Theorem, but under different conditions, namely for an i.i.d. random model with a common steady state $\pi>0$ in expectation and weak feedback property. While the assumption $\pi>0$ is stronger (not required in [15]), the weak feedback assumption is weaker than the requirement of almost sure positive diagonal entries of [15]. To see this, consider for example the timehomogeneous static model given by

$$
A(k)=\left[\begin{array}{ccc}
0 & \frac{1}{2} & \frac{1}{2} \\
\frac{1}{2} & 0 & \frac{1}{2} \\
\frac{1}{2} & \frac{1}{2} & 0
\end{array}\right] \quad \text { for } k \geq 0
$$

It can be seen that this model has weak-feedback property with $\gamma=1 / 4$, while all the diagonal entries are equal to zero. Hence, the ergodicity of the model cannot be deduced from [15]. However, since $\{A(k)\}$ has infinite flow property, by Infinite Flow Theorem it follows that the chain is ergodic.

Another consequence of the Infinite Flow Theorem, which cannot be (directly) deduced from the known results on i.i.d. and stationary random processes, is its application to the doubly stochastic deterministic chains. As discussed in Section II, any deterministic chain can be viewed as an independent random model. Therefore, the following result holds.

Corollary 1: Let $\{A(k)\}$ be a doubly stochastic deterministic chain with $A_{i i}(k) \geq \gamma$ for some $\gamma>0$ and all $i \in[m]$, $k \geq 0$. Then, $\{A(k)\}$ is ergodic if and only if $\{A(k)\}$ has infinite flow property.

We note that in Corollary 1, the doubly stochastic assumption can be replaced by $\pi^{T} A(k)=\pi^{T}$ for all $k \geq 0$ and a $\pi>0$.

As yet another corollary of the Infinite Flow Theorem, we provide an alternative characterization of random ergodic models by means of the infinite flow graph. Such a graph is known to be closely related to the ergodicity of deterministic chains from the seminal work of Tsitsiklis [18]. For a given random model $\{W(k)\}$, let its infinite flow graph be the undirected graph with the vertex set $[m]$ and the edge set

$$
\mathcal{E}^{\infty}=\left\{\{i, j\} \mid \sum_{k=0}^{\infty} W_{i j}(k)+W_{j i}(k)=\infty \text { a.s., } i \neq j\right\} .
$$

Based on the definition of the infinite flow graph, we have the following corollary of the Infinite Flow Theorem.

Corollary 2: Let the random model $\{W(k)\}$ have the steady state $\pi>0$ in expectation and the weak feedback property. Then, the model is ergodic if and only if its infinite flow graph is connected.

Proof: Let $G^{\infty}$ be the infinite flow graph of the given random model. Note that $G^{\infty}$ is not connected if and only if there exists a nontrivial vertex set $S \subset[m]$ such that there is no edge between $S$ and $\bar{S}$ in $G^{\infty}$. This holds if and only if $\sum_{k=0}^{\infty} \sum_{i \in S, j \in \bar{S}}\left(W_{i j}(k)+W_{j i}(k)\right)=\sum_{k=0}^{\infty} W_{S}(k)<\infty$, which is true if and only if the model does not have the infinite flow property. By Infinite Flow Theorem 4, this holds if and only if the model is not ergodic.

\section{IMPLICATIONS}

In this section we discuss the application of Infinite Flow Theorem 4 to extend the randomized gossip algorithm [2] as well as to study the network models with link failures. Then, we consider equal-neighbor average schemes on Erdös-Rényi type random graphs. Finally, we develop an alternative proof of the second Borel-Cantelli lemma by uncovering a relation between an infinite often event of a given sequence of events and the ergodicity of a certain chain.

\section{A. Application to Gossip Algorithm}

We discuss an application of our main result to extend the randomized gossip algorithm for a network with dynamic topology. The gossip algorithm has been originally proposed and analyzed in [2], [3] for a network with a static underlying connectivity topology.

We consider a network of $m$ agents which we view as nodes of a graph with the node set $[\mathrm{m}]$. Suppose that each agent has a private scalar value $x_{i}(0)$ at time $k=0$. Now, let the interactions of the agents be random at nonnegative integer valued time instances ${ }^{1} k$ as follows: At any time $k \geq$ 1 , two different agents $i, j \in[\mathrm{m}]$ wake up with probability $P_{i j}(k)$, where $P_{i j}(k)=P_{j i}(k)$ and $\sum_{i<j} P_{i j}(k)=1$. Then, they set their values to the average of their current values, i.e., $x_{i}(k)=x_{j}(k)=\frac{1}{2}\left(x_{i}(k-1)+x_{j}(k-1)\right)$. We assume that the choices of the pairs $\{i, j\}$ of interacting agents at different time instances are independent.

Based on the above agent interaction model, let us define the independent random model $\{W(k)\}$ by:

$$
W(k)=I-\frac{1}{2}\left(e_{i}-e_{j}\right)\left(e_{i}-e_{j}\right)^{T} \text { with probability } P_{i j}(k) .
$$

Then, the dynamic system (1) driven by the random chain $\{W(k)\}$ describes the evolution of the vector $x(k)$ that has its $i$-th component value equal to the agent value $x_{i}(k)$. Note that the model $\{W(k)\}$ in (6) is symmetric and hence, by the Infinite Flow Theorem we can immediately deduce the following result.

Lemma 4: For the extended gossip algorithm (6), the consensus is almost sure if $\sum_{k=0}^{\infty} P_{S}(k)=\infty$ for any nontrivial set $S \subset[m]$.

Proof: From (6), for any $i \in[m], W_{i i}(k)$ is equal to $\frac{1}{2}$ or 1 depending on whether a communication link between agent $i$ and some other agent $j$ is active at a given time $k$ or not. Therefore, the extended gossip scheme (6) is a random independent symmetric model with weak feedback property. Hence, as a result of Infinite Flow Theorem 4 and the relation $\mathrm{E}\left[W_{i j}(k)+W_{j i}(k)\right]=P_{i j}(k)$ for all $i \neq j$ and $k \geq 0$, it follows that the model is ergodic if and only if

$$
\sum_{k=0}^{\infty} P_{S}(k)=\sum_{k=0}^{\infty} \sum_{i \in S, j \in \bar{S}} P_{i j}(k)=\infty .
$$

Thus, the model admits consensus.

\footnotetext{
${ }^{1}$ Typically defined by a virtual Poisson clock, for more details see [3].
} 
Now, let us consider the infinite flow graph of the extended gossip model. Note that the model has weak feedback property, as discussed in the proof of Lemma 4. Thus, the model satisfies the conditions of Corollary 2 and, therefore, the extended gossip model is ergodic if and only if its infinite flow graph is connected. Its infinite flow graph consists of the edges $\{i, j\}$ that carry infinite flow, or equivalently in this case: the edges $\{i, j\}$ such that $\sum_{k=0}^{\infty} P_{i j}(k)=\infty$. Since the model is independent and $P_{i j}(k)$ is the probability that the communication between agents $i$ and $j$ is active at time $k$, by the second Borel-Cantelli lemma it follows that $\sum_{k=0}^{\infty} P_{i j}(k)=\infty$ if and only if the communication between agents $i$ and $j$ happens infinitely often. Therefore, we have the following corollary.

Corollary 3: For an extended gossip model $\{W(k)\}$, an edge $\{i, j\}$ belongs to its infinite flow graph $G^{\infty}$ if and only if agents $i$ and $j$ communicate with each other infinitely many times. Furthermore, the model is ergodic if and only if its infinite flow graph $G^{\infty}$ is connected.

In the original randomized gossip algorithm of [2], [3], the underlying connectivity topology is fixed. Let the topology be represented with an edge set $\mathcal{E}$. At any time $k$, a node is chosen uniformly from the set $[m]$ and it communicates with one of its neighbors $j$ with probability $Q_{i j}$, where $Q$ is a stochastic matrix and $Q_{i j}>0$ if and only if $\{i, j\} \in \mathcal{E}$. The update rule at each iteration is the same as in Eq. (6). Therefore, agents $i$ and $j$ communicate at time $k$ with probability $P_{i j}(k)=\frac{1}{m}\left(Q_{i j}+Q_{j i}\right)$. Hence, the sufficient condition of Lemma 4 for consensus reduces to the condition that the underlying network $([m], \mathcal{E})$ is connected. In this case, the infinite flow graph of the model is nothing but the underlying connectivity graph $([m], \mathcal{E})$ itself.

Using the same methodology, one can extend the broadcast algorithm [1] to the networks with dynamic topology.

\section{B. Link Failure Models}

Suppose that we are given a random model $\{W(k)\}$ with common steady state $\pi>0$ in expectation and weak feedback property. The index set $[m]$ is viewed as a set of agents and each $W(k)$ represents the weights that the agents use including the communication pattern among the agents at time $k$. Specifically, if $W_{i j}(k)(\omega)>0$ at a time $k$ for a realization $\omega$ and $i \neq j$, then there is a directed communication link $(j, i)$ from agent $j$ to agent $i$. Now, suppose that the links are not perfectly reliable, i.e., there exists a binary random variable $F_{i j}(k)$ at time $k$ such that the link $(j, i)$ fails if $F_{i j}(k)=1$ for $i \neq j$. Therefore, link failure pattern at time $k$ can be viewed as a random binary matrix $F(k)=\left[F_{i j}(k)\right]_{i, j \in[m]}$ with its diagonal entries equal to zero. We refer to the process $\{F(k)\}$ as the link failure process.

Based on a given random model $\{W(k)\}$ and link failure process $\{F(k)\}$, we are interested in the ergodicity of a new random model $\{U(k)\}$ which is defined as follows:

$$
U(k)=W(k) \cdot\left(e e^{T}-F(k)\right)+\operatorname{diag}([W(k) \cdot F(k)] e)
$$

where the dot operation "." represents the element-wise product of matrices and $\operatorname{diag}(v)$ is a diagonal matrix with its diagonal entries corresponding to the vector $v$ entries. The term $\operatorname{diag}([W(k) \cdot F(k)] e)$ is the compensation matrix for the link failures in the following sense: when a link $(j, i)$ fails, then from agent $i$ 's viewpoint, it seems that there was originally no link from the agent $j$ at time $k$. Therefore, agent $i$ will increase its self-feedback weight $W_{i i}(k)$ by $W_{i j}(k)$, which is the increase by $[W(k) \cdot F(k) e]_{i}$ in general. We refer to the model $\{U(k)\}$ as the link failure model. We consider following assumptions on the link failure model.

Assumption 1: Let $\{F(k)\}$ be a random model such that the following hold:

(a) $F_{i j}(k)$ is a binary random variable for all $k \geq 0$ and $i, j \in[m]$.

(b) The failure process $\{F(k)\}$ is an independent process.

(c) The random variables $\left\{F_{i j}(k) \mid i, j \in[m], i \neq j\right\}$ are i.i.d. for any $k \geq 0$.

Our next result is for the models with feedback property, defined as follows: a random model $\{W(k)\}$ is said to have feedback property if there exists $\gamma>0$ such that $\mathrm{E}\left[W_{i i}(k) W_{i j}(k)+W_{j j}(k) W_{j i}(k)\right] \geq \gamma \mathrm{E}\left[W_{i j}(k)+W_{j i}(k)\right]$ for any $k \geq 0$ and $i, j \in[m]$ with $i \neq j$. This condition is more restrictive than weak feedback property, but is still general enough to contain the i.i.d. models with positive diagonal elements and models with diagonal elements uniformly bounded from zero a.s. We have the following result.

Lemma 5: Let $\{W(k)\}$ be an independent model with common steady state $\pi>0$ in expectation and feedback property. Let $\{F(k)\}$ be a binary process satisfying Assumption 1. Also, let $\{W(k)\}$ and $\{F(k)\}$ be independent. Then, the failure model $\{U(k)\}$ is ergodic if and only if

$$
\sum_{k=0}^{\infty}\left(1-p_{k}\right) W_{S}(k)=\infty \quad \text { for all } S \subset[m],
$$

where $p_{k}=\operatorname{Pr}\left(F_{i j}(k)=1\right)$.

Proof: By the definition of the failure model $\{U(k)\}$ in (7), since both random processes $\{W(k)\}$ and $\{F(k)\}$ are independent, the failure model $\{U(k)\}$ is also independent. Then, for $i \neq j$ and for any $k \geq 0$, we have

$$
\mathrm{E}\left[U_{i j}(k)\right]=\mathrm{E}\left[W_{i j}(k)\left(1-F_{i j}(k)\right)\right]=\left(1-p_{k}\right) \mathrm{E}\left[W_{i j}(k)\right],
$$

where the last equality holds since $W_{i j}(k)$ and $F_{i j}(k)$ are independent, and $\mathrm{E}\left[F_{i j}(k)\right]=p_{k}$. The matrix $U(k)$ is surely stochastic, so that $1-\mathrm{E}\left[U_{i i(k)}\right]=\sum_{j \neq i} \mathrm{E}\left[U_{i j}(k)\right]$. By the preceding relation, we have $\sum_{j \neq i} \mathrm{E}\left[U_{i j}(k)\right]=(1-$ $\left.p_{k}\right) \sum_{j \neq i} \mathrm{E}\left[W_{i j}(k)\right]$, which by stochasticity of $W(k)$ implies $\sum_{j \neq i} \mathrm{E}\left[U_{i j}(k)\right]=\left(1-p_{k}\right)\left(1-\mathrm{E}\left[W_{i i}(k)\right]\right)$. Therefore, $\mathrm{E}\left[U_{i i}(k)\right]=p_{k}+\left(1-p_{k}\right) \mathrm{E}\left[W_{i i}(k)\right]$, or in matrix notation:

$$
\mathrm{E}[U(k)]=p_{k} I+\left(1-p_{k}\right) \mathrm{E}[W(k)],
$$

where $I$ denotes the identity matrix. But since $\pi$ is a common steady state of $\{\mathrm{E}[W(k)]\}$, it follows

$$
\pi^{T} \mathrm{E}[U(k)]=p_{k} \pi^{T}+\left(1-p_{k}\right) \pi^{T}=\pi^{T} .
$$


Hence, the model $\{U(k)\}$ has the same common steady state $\pi>0$ in expectation as the original model $\{W(k)\}$.

We next show that the failure model has feedback property. By the definition of $U(k)$, we have $U_{i i}(k) \geq W_{i i}(k)$ for all $i \in[m]$ and $k \geq 0$. Hence, $\mathrm{E}\left[U_{i i}(k) U_{i j}(k)\right] \geq$ $\mathrm{E}\left[W_{i i}(k) U_{i j}(k)\right]$. Since $\{F(k)\}$ and $\{W(k)\}$ are independent, we have

$$
\begin{aligned}
\mathrm{E}\left[W_{i i}(k) U_{i j}(k)\right] & =\mathrm{E}\left[\mathrm{E}\left[W_{i i}(k) U_{i j}(k) \mid F_{i j}(k)=0\right]\right] \\
& =\mathrm{E}\left[\mathrm{E}\left[W_{i i}(k) W_{i j}(k) \mid F_{i j}(k)=0\right]\right] \\
& =\left(1-p_{k}\right) \mathrm{E}\left[W_{i i}(k) W_{i j}(k)\right] .
\end{aligned}
$$

A similar relation holds for $\mathrm{E}\left[U_{j j}(k) U_{j i}(k)\right]$. Using the feedback property of $\{W(k)\}$, we have

$$
\begin{aligned}
& \mathrm{E}\left[U_{i i}(k) U_{i j}(k)+U_{j j}(k) U_{j i}(k)\right] \\
& \geq\left(1-p_{k}\right) \gamma \mathrm{E}\left[W_{i j}(k)+W_{j i}(k)\right] \\
& =\gamma \mathrm{E}\left[U_{i j}(k)+U_{j i}(k)\right],
\end{aligned}
$$

where the last equality follows from Eq. (8) and $\gamma>0$ is the feedback constant for $\{W(k)\}$. Thus, the failure model $\{U(k)\}$ has feedback property with constant $\gamma$. Hence, the model satisfies the assumptions of Infinite Flow Theorem 4. By this theorem, the model $\{U(k)\}$ is ergodic if and only if $\sum_{k=0}^{\infty} \mathrm{E}\left[U_{S}(k)\right]=\infty$ for any nontrivial $S \subset[m]$. By Eq. (8) we have $\mathrm{E}\left[U_{S}(k)\right]=\left(1-p_{k}\right) \mathrm{E}\left[W_{S}(k)\right]$. Hence, the failure model $\{U(k)\}$ is ergodic if and only if $\sum_{k=0}^{\infty}(1-$ $\left.p_{k}\right) \mathrm{E}\left[W_{S}(k)\right]=\infty$ for any nontrivial $S \subset[m]$.

When the failure probabilities $p_{k}$ are uniformly bounded away from one, i.e., $p_{k} \leq \bar{p}$ for all $k$ and some $\bar{p}<1$, we have that $\sum_{k=0}^{\infty}\left(1-p_{k}\right) \mathrm{E}\left[W_{S}(k)\right]=\infty$ if and only if $\sum_{k=0}^{\infty} \mathrm{E}\left[W_{S}(k)\right]=\infty$. Thus, when the failure probabilities $p_{k}$ are uniformly bounded away from one, by Lemma 5 we have: the failure model $\{U(k)\}$ is ergodic if and only if the original model $\{W(k)\}$ is ergodic.

Remark 1: The condition of Assumption 1 that $F_{i j}(k)$ is a binary random variable can be replaced with the condition that $F_{i j}(k)$ is a continuous random variable taking values in the interval $[0,1]$. In this case, $\left(1-F_{i j}(k)\right) W_{i j}(k)$ represents a randomly attenuated link instead of a failed link, and Lemma 5 applies to a randomly attenuated model. This can be shown by using almost the same proof as for Lemma 5 .

\section{Equal-Neighbor Average on Random Graphs}

In the Erdös-Rényi random model, a random graph $\mathcal{G}([m], p)$ is the graph defined on the vertex set $[m]$ with edges selected at random with a probability value $p>0$. The random graph $\mathcal{G}(m, p)$ is constructed by selecting an edge $\{i, j\}$ from the complete $\operatorname{graph}^{2}$ with probability $p$ independently of the other edges (see, for example, [5]).

Here, we will consider random directed subgraphs of a given directed regular graph, denoted by $G=([m], \mathcal{E})$. Let a random (directed) graph $R(G, p)$ be the graph on the vertex set $[m]$ with the edge set obtained by selecting each edge from $\mathcal{E}$ with probability $p$ independently of the other edges. Consider a sequence of independent random graphs

\footnotetext{
${ }^{2} \mathrm{~A}$ graph is complete if there is an edge between any two of its vertices.
}

$\left\{R\left(G_{k}, p_{k}\right)\right\}$, where $G_{k}=\left([m], \mathcal{E}_{k}\right)$ and $p_{k} \in[0,1]$ for all $k \geq 0$. Let $G_{c}=\left([m], \mathcal{E}_{c}\right)$ be the graph with the edge set $\mathcal{E}_{c}$ consisting of the edges that appear infinitely often in the edge sequence $\left\{\mathcal{E}_{k}\right\}$ of the graphs $\left\{R\left(G_{k}, p_{k}\right)\right\}$. Since the sequence $\left\{\mathcal{E}_{k}\right\}$ is independent, the set $\mathcal{E}_{c}$ is a tale event. Hence, by Kolmogorov's 0-1 law [7], $\mathcal{E}_{c}$ is a trivial event. Therefore, the graph $G_{c}$ is almost surely one of the $2^{m(m-1)}$ possible directed graphs on $[m]$. We say the graph $G_{c}$ is the connectivity graph of the sequence $\left\{R\left(G_{k}, p_{k}\right)\right\}$.

Let $G=([m], \mathcal{E})$ be a directed graph. Define the equalneighbor weight matrix $U(G)$ on $G$ as the matrix with $U_{i j}(G)=\frac{1}{N_{i}^{+}(G)+1}$ for $(i, j) \in \mathcal{E}$ or $j=i$, where $N_{i}^{+}(G)$ is the number of in-neighbors of node $i$, i.e., $N_{i}^{+}(G)=\mid\{j \in$ $[m] \mid(j, i) \in \mathcal{E}\} \mid$ for any $i \in[m]$. We view the vertices of the graph as agents, and assume that each of them has private scalar $x_{i}$. Then, $[U(G) x]_{i}$ is the value of agent $i$ obtained by averaging uniformly its value with the values $x_{j}$ from its inneighbors. We also use the number $N_{i}^{-}(G)$ of out-neighbors of a node $i$, i.e., $N_{i}^{-}(G)=|\{j \in[m] \mid(i, j) \in \mathcal{E}\}|$. We have the following result for directed graphs with the same in- and out-degrees at each node.

Lemma 6: Let $\left\{G_{k}\right\}$ be a sequence of directed graphs with $G_{k}=\left([m], \mathcal{E}_{k}\right)$ such that $N_{i}^{+}\left(G_{k}\right)=N_{i}^{-}\left(G_{k}\right)$ for all $i \in[m]$ and $N_{i}^{+}\left(G_{k}\right)=N_{j}^{+}\left(G_{k}\right)$ for all $i, j \in[m]$ and all $k \geq 0$. Let $\left\{R\left(G_{k}, p_{k}\right)\right\}$ be its related sequence of independent random graphs where $p_{k} \in[0,1]$ for all $k \geq 0$. Then, the equal-neighbor matrix sequence $\left\{U\left(R\left(G_{k}, p_{k}\right)\right)\right\}$ is ergodic if and only if the connectivity graph of $\left\{R\left(G_{k}, p_{k}\right)\right\}$ is connected $^{3}$. In particular, if each $G_{k}$ is connected, then $\left\{U\left(R\left(G_{k}, p_{k}\right)\right)\right\}$ is ergodic if and only if $\sum_{k=0}^{\infty} p_{k}=\infty$.

Proof: Let $W(k)=U\left(R\left(G_{k}, p_{k}\right)\right)$ for all $k$. The distribution of $W_{i i}(k)$ is just a function of $p_{k}$ and $N_{i}^{+}\left(G_{k}\right)$. Since $N_{i}^{+}\left(G_{k}\right)=N_{j}^{+}\left(G_{k}\right), \mathrm{E}\left[W_{i i}(k)\right]$ is independent of the index $i$. Also, note that the distributions of $W_{i j}(k)$ and $W_{i \ell}(k)$ are the same for any two edges $(i, j),(i, \ell) \in \mathcal{E}_{k}$. Thus, by the stochasticity of $\mathrm{E}[W(k)]$, we obtain $\mathrm{E}\left[W_{i j}(k)\right]=\frac{1-\mathrm{E}\left[W_{i i}(k)\right]}{N_{i}^{+}\left(G_{k}\right)}$ which is independent of the index $i$. Hence, $\mathrm{E}\left[W_{i_{1} j_{1}}(k)\right]=$ $\mathrm{E}\left[W_{i_{2} j_{2}}(k)\right]$ for any $\left(i_{1}, j_{1}\right),\left(i_{2}, j_{2}\right) \in \mathcal{E}_{k}$. Since $N_{i}^{+}\left(G_{k}\right)=$ $N_{i}^{-}\left(G_{k}\right)$ for any $i$, the expected model $\{\mathrm{E}[W(k)]\}$ has a common steady state $\frac{1}{m} e$. Note that $W_{i i}(k) \geq \frac{1}{m}$ for all $i \in[m]$ and $k$, implying that the model has weak feedback property with $\gamma=\frac{1}{m}$. Thus, by Infinite Flow Theorem 4, the model is ergodic iff $\sum_{k=0}^{\infty} W_{S}(k)=\infty$ for any $S \subset[m]$.

We now show that $\sum_{k=0}^{\infty} W_{S}(k)=\infty$ for any $S \subset[m]$ is equivalent to the connectivity graph $G_{c}$ of $\left\{R\left(G_{k}, p_{k}\right)\right\}$ being connected. For $S \subset[m]$ and $k \geq 0$, define $\chi_{S}(k)=1$ if there are edges between $S$ and $\bar{S}$ in $R\left(G_{k}, p_{k}\right)$, and otherwise $\chi_{S}(k)=0$. By the definition of the equal-neighbor dynamics, if $W_{i j}(k)>0$ then $W_{i j}(k) \geq \frac{1}{m}$, implying $\frac{1}{m} \chi_{S}(k) \leq W_{S}(k) \leq m \chi_{S}(k)$. Thus, $\sum_{k=0}^{\infty} W_{S}(k)=\infty$ if and only if $\sum_{k=0}^{\infty} \chi_{S}(k)=\infty$, which holds if and only if $S$ and $\bar{S}$ are connected infinitely often. Therefore, $\sum_{k=0}^{\infty} W_{S}(k)=\infty$ if and only if there is an edge $(i, j)$ such that $i \in S, j \in \bar{S}$ and $W_{i j}(k)>0$ infinitely often, which is equivalent to the connectivity graph $G_{c}$ being connected.

\footnotetext{
${ }^{3}$ There exists an undirected path between any two nodes in the graph.
} 
When the graph $G_{k}$ is connected, the probability of having edges between $S$ and $\bar{S}$ in $R\left(G_{k}, p_{k}\right)$ is bounded below by $p_{k}$ and above by $2|S||\bar{S}| p_{k}$. Since the model is independent, by the Borel-Cantelli lemmas [7], the sets $S$ and $\bar{S}$ are connected infinitely often if and only if $\sum_{k=0}^{\infty} p_{k}=\infty$.

Consider an instance of Lemma 6 where each $G_{k}$ is the complete directed graph over the set $[m]$ for $m>2$. Then, the consensus is achieved iff $\sum_{k=0}^{\infty} p_{k}=\infty$. This is true, for example, when $p_{k}=\frac{1}{\alpha+k}$ for some $\alpha>>m^{2}$, which results in (most probably) sparse graphs $R\left(G_{k}, p_{k}\right)$. Let $R_{k}^{k+B}$ be the random graph defined as the union of graphs $R\left(G_{k}, p_{k}\right), \ldots, R\left(G_{k+B-1}, p_{k+B-1}\right)$, where $B \geq 1$ and $k \geq 0$. The probability of having two edges in $R_{k}^{k+\bar{B}}$ is bounded above by $\left(B m p_{k}\right)^{2}$ which is summable. Hence, by the first Borel-Cantelli lemma [7], the probability of having two edges in $R_{k}^{k+B}$ infinitely often is zero. This implies that not only the probability of having $R_{k}^{k+B}$ connected goes to zero but also the probability of having $R_{k}^{k+B}$ connected infinitely often is zero.

\section{Alternative proof of Borel-Cantelli lemma}

Here, we discuss an alternative proof of the second BorelCantelli lemma [7]. For a sequence of events $\left\{E_{k}\right\}$ in some probability space, let us define a sequence of random $2 \times 2$ stochastic matrices $\{W(k)\}$ as follows: $W(k)=\frac{1}{2} e e^{T} \mathbf{1}_{E_{k}}+$ $I 1_{E^{c}}$ for $k \geq 0$, i.e., $W(k)$ is equal to the stochastic matrix $\frac{1}{2} e e^{T}$ on $E_{k}$ and otherwise, it is equal to the identity matrix.

Let $U=\cap_{k=0}^{\infty} \cup_{s=k}^{\infty} E_{s}=\left\{E_{k} i\right.$ i.o. $\}$ where i.o. stands for infinitely often. Then, $\omega \in U$ means that in the corresponding random chain $\{W(k)\}(\omega)$ we have $W(k)(\omega)=\frac{1}{2} e^{T} e$ for infinitely many indices $k$. Let $\{A(k)\}$ be a chain of $2 \times 2$ matrices where $A(k)$ is either $I$ or $\frac{1}{2} e e^{T}$ for all $k \geq 0$. Since $M\left(\frac{1}{2} e e^{T}\right)=\frac{1}{2} e e^{T}$ for any $2 \times 2$ stochastic matrix $M$, it follows that the chain $\{A(k)\}$ is ergodic if and only if the matrix $\frac{1}{2} e e^{T}$ appears infinitely many times in the chain. This observation, together with Infinite Flow Theorem, gives rise to an alternative proof of the second Borel-Cantelli lemma.

Theorem 5: Second Borel-Cantelli Lemma: Let $\left\{E_{k}\right\}$ be independent and $\sum_{k=0}^{\infty} \operatorname{Pr}\left(E_{k}\right)=\infty$. Then $\operatorname{Pr}(U)=1$.

Proof: Let $\{W(k)\}$ be the random chain corresponding to the sequence $\left\{E_{k}\right\}$. Then $\{W(k)\}(\omega)$ is ergodic if and only if $\omega \in U$. Since $\sum_{k=0}^{\infty} \operatorname{Pr}\left(E_{k}\right)=\infty$, it follows $\sum_{k=0}^{\infty} \mathrm{E}\left[W_{12}(k)+W_{21}(k)\right]=\infty$, implying that $\{W(k)\}$ has infinite flow. Furthermore, since $\left\{E_{k}\right\}$ is independent, so is $\{W(k)\}$. Observe that each realization of $W(k)$ is doubly stochastic; hence, $\pi=\frac{1}{2} e$ is the common steady state for $\{\mathrm{E}[W(k)]\}$. We also have $W_{i i}(k) \geq \frac{1}{2}$ for $i=1,2$ and any $k$. Therefore, by Infinite Flow Theorem 4, the model $\{W(k)\}$ is ergodic and, hence, $\operatorname{Pr}(U)=1$.

In the derivation of the above proof, there is a possibility of being exposed to the trap of circular reasoning. But to the best of authors' knowledge, none of the steps toward Infinite Flow Theorem involves the use of this result.

\section{CONCLUSION}

We have studied the ergodicity and consensus of timeinhomogeneous random dynamic system driven by stochastic matrices. We achieved this through the light of an important property which we termed infinite flow property. We showed that the infinite flow property is necessary for the ergodicity of any random model and that it is sufficient for a general class of independent random models. Then, we have shown that, for a class of random independent models, the ergodicity of the model and the ergodicity of the expected model are equivalent, as stated in Infinite Flow Theorem. Using this theorem, we have analyzed extensions of randomized gossip algorithm and derived a necessary and sufficient condition for ergodicity of independent link failure models. We have also established a necessary and sufficient condition for the convergence of equal-neighbor average algorithms.

\section{APPENDIX}

\section{REFERENCES}

[1] T.C. Aysal, M.E. Yildriz, A. D. Sarwate, and A. Scaglione, Broadcast gossip algorithms for consensus, IEEE Transactions on Signal processing 57 (2009), 2748-2761.

[2] S. Boyd, A. Ghosh, B. Prabhakar, and D. Shah, Gossip algorithms: Design, analysis, and applications, Proceedings IEEE Infocom, 2005, pp. 1653-1664.

[3] _ Randomized gossip algorithms, IEEE Transactions on Information Theory $\mathbf{5 2}$ (2006), no. 6, 2508-2530.

[4] S. Chatterjee and E. Seneta, Towards consensus: Some convergence theorems on repeated averaging, Journal of Applied Probability 14 (1977), no. 1, 89-97.

[5] F. Chung and L. Lu, Complex graphs and networks, American Mathematical Society, Providence, Rhode Island, USA, 2006.

[6] R. Cogburn, On products of random stochastic matrices, In Random matrices and their applications (1986), 199-213.

[7] R. Durrett, Probability: Theory and examples, third ed., Curt Hinrichs, 2005.

[8] F. Fagnani and S. Zampieri, Randomized consensus algorithms over large scale networks, IEEE Journal on Selected Areas in Communications 26 (2008), no. 4, 634-649.

[9] I. Lobel and A. Ozdaglar, Distributed subgradient methods over random networks, Tech. report, Laboratory for Information and Decision Systems, Report 2800, MIT, 2008.

[10] K. Nawrotzki, Discrete open systems on markov chains in a random environment. I, Elektronische Informationsverarbeitung und Kybernetik 17 (1981), 569-599.

[11] Discrete open systems on markov chains in a random environment. II, Elektronische Informationsverarbeitung und Kybernetik 18 (1982), 83-98.

[12] A. Nedić, A. Olshevsky, A. Ozdaglar, and J.N. Tsitsiklis, On distributed averaging algorithms and quantization effects, to appear in IEEE Transactions on Automatic Control, 2009.

[13] A. Olshevsky and J.N. Tsitsiklis, Convergence speed in distributed consensus and averaging, SIAM Journal on Control and Optimization 48 (2008), no. 1, 33-55.

[14] M. Rosenblatt, Products of independent identically distributed stochastic matrices, Journal of Journal of Mathematical Analysis and Applications 11 (1965), no. 1, 1-10.

[15] A. Tahbaz-Salehi and A. Jadbabaie, A necessary and sufficient condition for consensus over random networks, IEEE Transactions on Automatic Control 53 (2008), no. 3, 791-795.

[16] Consensus over ergodic stationary graph processes, to appear in IEEE Transactions on Automatic Control, 2009.

[17] B. Touri and A. Nedić, On ergodicity, infinite flow and consensus in random models, available at http://arxiv.org/abs/1001.1890.

[18] J.N. Tsitsiklis, Problems in decentralized decision making and computation, Ph.D. thesis, Dept. of Electrical Engineering and Computer Science, MIT, 1984.

[19] J.N. Tsitsiklis, D.P. Bertsekas, and M. Athans, Distributed asynchronous deterministic and stochastic gradient optimization algorithms, IEEE Transactions on Automatic Control 31 (1986), no. 9, 803-812. 